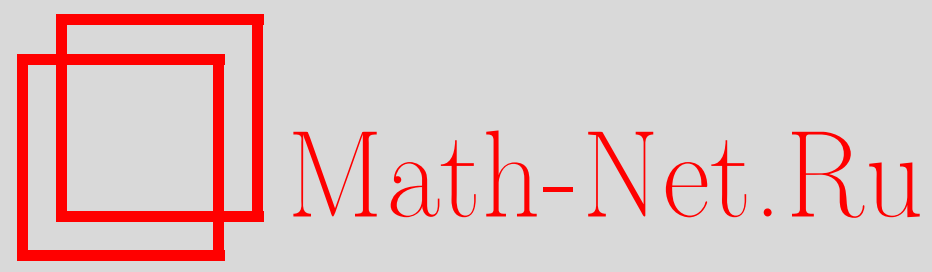

А. Б. Еловиков, Факторизация однопорожденных формаций, Матем. заметки, 2003, том 73, выпуск 5, 684-697

DOI: https://doi.org/10.4213/mzm215

Использование Общероссийского математического портала Math-Net.Ru подразумевает, что вы прочитали и согласны с пользовательским соглашением http://www.mathnet.ru/rus/agreement

Параметры загрузки:

IP: 54.210 .77 .194

26 апреля 2023 г., 13:37:18

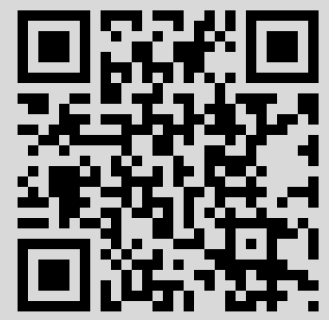




\title{
ФАКТОРИЗАЦИЯ ОДНОПОРОЖДЕННЫХ ФОРМАЦИЙ
}

\author{
А. Б. Еловиков
}

\begin{abstract}
В настоящей работе описан широкий класс однопорожденных факторизуемых расслоенных формаций, включающий в себя композиционные, биканонические и другие виды формаций. В. А. Ведерниковым было предложено с единых позиций изучать расслоенные формации с направлениями $\beta, \alpha \leqslant \beta \leqslant \gamma$, где $\alpha$ и $\gamma$ также некоторые направления. В статье исследуются однопорожденные факторизуемые расслоенные формации с направлениями из отрезка $[\alpha, \gamma]$, где $\alpha, \gamma$ - направления биканонической и композиционной формаций соответственно. В частности, изучены все несократимые факторизации однопорожденных композиционных формаций.
\end{abstract}

Библиография: 9 названий.

Однопорожденные формации конечных групп были введены в рассмотрение основателем теории формаций В. Гашюцем в 1963 г. Исследованию факторизуемых однопорожденных локальных формаций посвящен ряд работ А.Н. Скибы, например, [1], [2]. В книге [3] Скиба описал все возможные факторизации однопорожденных локальных формаций. Там же был поставлен вопрос об описании всех несократимых факторизаций композишионных формаций [3, вопрос 3.5.21].

В работах [4], [5] В.А. Ведерниковым и М. М. Сорокиной была предложена концепция расслоенных формаций. Это позволило классифищировать уже известные формации и конструировать новые виды формаций с помощью функции направления.

В настоящей работе рассматривается широкий класс расслоенных формаций, включаюший в себя композищионные, биканонические и другие типы формаций. Описаны все несократимые факторизации однопорожденных расслоенных формаций данного класса, у которых первьй множитель также является расслоенной формацией. В частности, учитьвая предложение 3.5.21 [3], получен полный ответ на вопрос 3.5.21 из [3].

Рассматриваются только конечные группы. Все необходимые определения и обозначения можно найти в [3]-[5]. Приведем только некоторые из них. Через $G=[A] B$ обозначают полупрямое произведение групा $A$ и $B ;$ символами $\mathfrak{G}, \mathfrak{G} A, \mathfrak{I}, \mathfrak{S}, \mathfrak{S}_{c A}$ обозначают соответственно класс всех конечных групп, класс всех конечных $A$-групп, класс всех конечных простьх групп, класс всех конечных разрешимых групп, класс тех конечных групп, у которых все главные $A$-факторы центральны. Пусть $\mathfrak{F}$ и $\mathfrak{H}-$ формации. Тогда $\mathfrak{F H}$ обозначает формационное произведение $\mathfrak{F}$ и $\mathfrak{H}[4]$. Формация назьвается расслоенной с направлением $\varphi[4],[5]$, если $\mathfrak{F}=F(f, \varphi)=\left(G \in \mathfrak{G}: G / G_{\varphi(A)} \in f(A)\right.$ для всех $A \in K(G)$ ). Обозначим через $\alpha(\gamma)$ - направление биканонической (композиционной) формации, т.е. $\alpha(A)=\mathfrak{G}_{A^{\prime}}$ для любой неабелевой групшы $A \in \mathfrak{I}_{\text {и }} \alpha(A)=\mathfrak{G}_{A^{\prime}} \mathfrak{G}_{A}$ для 
любой абелевой групшы $A \in \mathfrak{I}\left(\gamma(A)=\mathfrak{S}_{c A}\right.$ для всех групп $\left.A \in \mathfrak{I}\right)$. Направление $\varphi$ расслоенной формации назьвают $b$-направлением, если $\varphi(A) \mathfrak{G}_{A}=\varphi(A)$ для любой абелевой групшы $A \in \mathfrak{I} ; r$-направлением, если $\varphi(A)=\mathfrak{G}_{A^{\prime}} \varphi(A)$ для любой $A \in \mathfrak{I}[4]$. На множестве $\Phi$ всех функций $\varphi: \mathfrak{I} \rightarrow$ (непустые формации Фиттинга) введено отношение частичного порядка $\leqslant$. Для любых $\varphi_{1}, \varphi_{2} \in \Phi$ полагают $\varphi_{1} \leqslant \varphi_{2}$, если $\varphi_{1}(A) \subseteq \varphi_{2}(A)$ для любой $A \in \mathfrak{I}[4]$.

Перейдем к изложению полученных результатов.

Используя методы, разработанные в [4], несложно показать, что выполняется

Лемма 1. Пусть $f_{1} u f_{2}$ - внутренние спутники расслоенной формации $\mathfrak{F} c$ br-направлением $\beta, \alpha \leqslant \beta \leqslant \gamma$. Тогда

1) $\mathfrak{G}_{A} f_{1}(A) \subseteq \mathfrak{F}$ для любого $A \in \mathfrak{I} \cap \mathfrak{A}$;

2) $\mathfrak{G}_{A} f_{1}(A)=\mathfrak{G}_{A} f_{2}(A)$ для любого $A \in \mathfrak{I} \cap \mathfrak{A}$;

3) $\mathfrak{F}$ обладает внутренним спутником $g$ таким, что $g(A)=\mathfrak{F}$, если $A \in \mathfrak{I} \backslash \mathfrak{A}$ $u g(A)=\mathfrak{G}_{A} f_{1}(A)$ для любого $A \in \mathfrak{I} \cap \mathfrak{A}$.

Из леммы 1 непосредственно вытекает

Лемма 2. Пусть $f$-внутренний спутник расслоенной формации $\mathfrak{F}$ с br-направлением $\beta, \alpha \leqslant \beta \leqslant \gamma$. Тогда формаиия $\mathfrak{F}$ имеет единственный максимальный внутренний спутник $h$, причем $h(A)=\mathfrak{F}$, если $A \in \mathfrak{I} \backslash \mathfrak{A} u h(A)=\mathfrak{G}_{A} h(A)=$ $\mathfrak{G}_{A} f(A)$ для любого $A \in \mathfrak{I} \cap \mathfrak{A}$.

Лемма 3. Пусть $f$-максимальный внутренний спутник расслоенной формаиии $\mathfrak{F}$ с br-направлением $\beta, \alpha \leqslant \beta \leqslant \gamma$. Если $\mathfrak{F}$ - наследственная формация, то $f(A)$ также является наследственной для всех $A \in \mathfrak{I}$.

ДокаЗАтЕльство. Допустим, что существует $A \in \mathfrak{I}$ такая, что найдется $G \in f(A)$, $M<G$ и $M \notin f(A)$, причем $G$ - группа минимального порядка с таким свойством. Тогда $G \neq 1$. Если $G$ - не монолитическая, то существуют две различные минимальные нормальные подгрупшы $R$ и $N$ групшы $G$, причем $G / R \in f(A)$ и $G / N \in f(A)$. По индукции $M R / R \in f(A)$ и $M N / N \in f(A)$, а значит $M /(M \cap R \cap N) \cong M \in f(A)$. Противоречие. Следовательно, $G$ - монолитическая групша с монолитом $R$ и $M R / R \in f(A)$. По лемме $2 f(A)=\mathfrak{F}$ для всех $A \in \mathfrak{I} \backslash \mathfrak{A}, f(A)=\mathfrak{G}_{A} f(A)$, если $A \in \mathfrak{A}$. Поэтому $A-$ абелева $p$-групша. Допустим, что $O_{A}(G) \neq 1$. Тогда $R \subseteq O_{A}(G)=O_{p}(G)$ для некоторого простого числа $p$. Но в этом случае $M \in \mathfrak{G}_{A} f(A)=f(A)$. Противоречие. Значит, $O_{A}(G)=1$.

По лемме 18.8 [6] существует точньй неприводимьй $F_{p}[G]$-модуль $T$. Рассмотрим $\Gamma=[T] G$. Поскольку $\Gamma / T \cong G \in f(A) \subseteq \mathfrak{F}$ и $O_{p}(\Gamma) \subseteq \Gamma_{\beta(A)}$, по лемме $2[4] \Gamma \in \mathfrak{F}$. Так как $T M<\Gamma$, по условию $T M \in \mathfrak{F}$. Тогда $T M /(T M)_{\beta(A)} \in f(A)$. Поскольку $C_{\Gamma}(T)=T=C_{T M}(T)-p$-группа, то $(T M)_{\gamma(A)}=F_{A}(T M)=O_{p}(T M)$. Значит, $(T M)_{\beta(A)}=O_{p}(T M)=O_{p}(T M) \cap T M=T\left(O_{p}(T M) \cap M\right) \subseteq T O_{p}(M)$. Таким образом, $T M /\left(T O_{p}(M)\right) \cong M /\left(M \cap T O_{p}(M)\right) \cong M /\left(O_{p}(M)(M \cap T)\right)=M / O_{p}(M) \in f(A)$ и $M \in \mathfrak{G}_{A} f(A)=f(A)$. Противоречие. В силу произвольности выбора $A f(A)$ - наследственная формация для всех $A \in \mathfrak{I}$. Лемма доказана.

Непустое множество формаций $\theta$ назьвается полной решеткой формащий, если $\varnothing$, $\mathfrak{G} \in \theta$ и пересечение любой совокупности формаций из $\theta$ снова принадлежит $\theta$ [7]. Через 
$\theta^{\beta}$ обозначим совокупность всех расслоенных формаций с направлением $\beta$, обладаюших $\theta$-значньп спутником.

ЛЕмма 4. Пусть $\theta$ - полная решетка формаиий $и \mathfrak{X}-$ непустой класс групп такой, что $\mathfrak{X} \subseteq \mathfrak{H} \in \theta^{\beta}$ u $\mathfrak{F}=\theta^{\beta} F(\mathfrak{X})$, дде $\varphi_{0} \leqslant \beta$. Тогда $\mathfrak{F}$ обладает единственным минимальным $\theta$-значным спутником $f$, причем $f(A)=\theta$ form $\left(G / G_{\beta(A)} \mid G \in \mathfrak{X}\right)$ для всех $A \in K(\mathfrak{X})$ u $f(A)=\varnothing$, если $A \in \mathfrak{I} \backslash K(\mathfrak{X})$.

Доказательство проводится аналогично теореме 5 в [5].

СЛЕДСТВИЕ 1. Пусть $f_{i}-$ минимальный $\theta$-значный спутник расслоенной формачии $\mathfrak{F}_{i}$ с br-направлением $\beta, \alpha \leqslant \beta \leqslant \gamma, i=1,2$. Тогда и только тогда $\mathfrak{F}_{1} \subseteq \mathfrak{F}_{2}$, когда $f_{1} \leqslant f_{2}$.

СЛЕДСТВИЕ 2. Пусть $f_{i}$ - максимальный внутренний $\theta$-значный спутник расслоенной формачии $\mathfrak{F}_{i}$ c br-направлением $\beta, \alpha \leqslant \beta \leqslant \gamma, i=1,2$. Тогда и только тогда $\mathfrak{F}_{1} \subseteq \mathfrak{F}_{2}, \kappa о г \partial а f_{1} \leqslant f_{2}$.

Через $F_{n}(\mathfrak{X}, \beta)$ будем обозначать $n$-кратно расслоенную формацию с направлением $\beta$, порожденную множеством групп $\mathfrak{X}$.

СледСТвИЕ 3. Пусть $\mathfrak{F}=F_{n}(\mathfrak{X}, \beta)$ с br-направлением $\beta, \alpha \leqslant \beta \leqslant \gamma, n \geqslant 1, \mathfrak{X}-$ некоторый класс әрупп. Тогда $K(\mathfrak{F})=K(\mathfrak{X})$.

ЛЕмма 5. В однопорожденной расслоенной формации $F(G, \beta)$ с бг-направлением $\beta, \alpha \leqslant \beta \leqslant \gamma$, содерэится лишь конечное множсество наследственных расслоенных формачий с направлением $\beta$.

ДокАЗАТЕЛЬСТво. Обозначим через $\theta$ полную решетку всех наследственных формаций. Пусть $\mathfrak{M}=\theta^{\beta} F(G)$ - пересечение всех расслоенных формаций с направлением $\beta$, содержащих $G$ и имеющих хотя бы один $\theta$-значный спутник. Очевидно, что $F(G, \beta) \subseteq \mathfrak{M}$. Покажем, что в $\mathfrak{M}$ содержится лишь конечное число наследственных расслоенных формаций с направлением $\beta$.

Пусть $\mathfrak{F}$ - произвольная наследственная расслоенная формация с направлением $\beta$ такая, что $\mathfrak{F} \subseteq \mathfrak{M}$. По лемме $3 \mathfrak{F}$ имеет хотя бы один $\theta$-значный спутник. Пусть $f$ и $m-$ минимальные наследственные спутники формаций $\mathfrak{F}$ и $\mathfrak{M}$ соответственно. Тогда по следствию $1 f \leqslant m$. По лемме $4 m(A)=\theta$ form $\left(G / G_{\beta(A)}\right)$ для всех $A \in K(G)$ и $m(A)=\varnothing$, если $A \in \mathfrak{I} \backslash K(G)$ - однопорожденные формации. Следовательно, по лемме 8.8 в [6] существует лишь конечное множество наследственных спутников $t<m$. Значит в $\mathfrak{M}$, а тем более в $F(G, \beta)$, существует лиш конечное множество наследственных расслоенных формаций с направлением $\beta$. Лемма доказана.

Лемма 6. Если $\mathfrak{F}=\mathfrak{M H}$, әде $\mathfrak{F} u \mathfrak{M}$ - неединичные расслоенные формации $с$ br-направлением $\beta, \alpha \leqslant \beta \leqslant \gamma$, a $\mathfrak{H}-$ такая формачия, что $K(\mathfrak{H}) \cap \mathfrak{A} \nsubseteq K(\mathfrak{M}) \cap \mathfrak{A} \neq$ $\varnothing$, то множество наследственных расслоенных подформаций с направлением $\beta$ формачии ғ бесконечно.

ДокАЗАТЕЛЬСТво. Пусть $Z_{p} \in K(\mathfrak{H}) \backslash K(\mathfrak{M})$. Ясно, что $\mathfrak{H} \subseteq \mathfrak{F}$. Значит $Z_{p} \in K(\mathfrak{F})$. По лемме $1 \mathfrak{N}_{p} \subseteq \mathfrak{F}$. Пусть $A$ - произвольная групша из $\mathfrak{N}_{p}$. Так как $A \in \mathfrak{F}=\mathfrak{M H}$, то $A^{\mathfrak{H}} \in \mathfrak{M}$. Но $Z_{p} \notin K(\mathfrak{M})$. Поэтому $A \in \mathfrak{H}$. Таким образом, $\mathfrak{N}_{p} \subseteq \mathfrak{H}$. Пусть $Z_{q} \in K(\mathfrak{M})$. 
Тогда $q \neq p$ и $\mathfrak{N}_{q} \subseteq \mathfrak{M}$. Для всякого натурального числа $n$ зафиксируем некоторую циклическую группу $P_{n}$ порядка $p^{n}$. Построим регулярное сплетение $Q_{n}=Z_{q}$ 々 $P_{n}$. Обозначим через $\mathfrak{F}_{n}$ расслоенную формацию с направлением $\beta$, порожденную групой $Q_{n}$. Группа $Q_{n} \in \mathfrak{N}_{q} \mathfrak{N}_{p} \subseteq \mathfrak{M H}=\mathfrak{F}$. Поэтому $\mathfrak{F}_{n} \subseteq \mathfrak{F}$. Поскольку группа $Q_{n}$ метанильпотентна, то $\mathfrak{F}_{n} \subseteq \mathfrak{N}^{2}$. Из доказательства теоремы 4 и следствия 4.1 [5] легко видеть, что каждая разрешимая расслоенная формация с $b r$-направлением $\beta, \alpha \leqslant \beta \leqslant \gamma$, является локальной формацией. Значит, ввиду леммы 8.10 [6] все расслоенные подформации с направлением $\beta$ формации $\mathfrak{N}^{2}$ наследственны. Тогда $\mathfrak{F}_{n}-$ наследственная расслоенная подформация в $\mathfrak{F}$.

При доказательстве леммы 8.13 [6] было показано, что если $n$ и $m$ - различные натуральные числа, то $\mathfrak{F}_{n} \neq \mathfrak{F}_{m}$. Итак, в $\mathfrak{F}$ содержится бесконечное множество наследственных расслоенных подформаций $\mathfrak{F}_{1}, \mathfrak{F}_{2}, \ldots$ с направлением $\beta$. Лемма доказана.

Лемма 7. Пусть $\mathfrak{M}=F(m, \beta)$ с br-направлением $\beta, \alpha \leqslant \beta \leqslant \gamma, u \mathfrak{H}-$ такая непустая формачия, что $K(\mathfrak{H}) \cap \mathfrak{A} \subseteq K(\mathfrak{M})$. Тогда $\mathfrak{F}=\mathfrak{M H}-$ расслоенная формачия с направлением $\beta$, причем она обладает таким спутником $f$, что $f(A)=m(A) \mathfrak{H}$ для всех $A \in K(\mathfrak{M}) ; f(A)=\mathfrak{H}$, если $A \in K(\mathfrak{H}) \backslash K(\mathfrak{M})$ u $f(A)=\varnothing$ npu $A \in \mathfrak{I} \backslash(K(\mathfrak{H}) \cup K(\mathfrak{M}))$.

ДокАЗАТЕЛЬСТво. Пусть $\mathfrak{F}_{1}=F(f, \beta)$. Покажем, что $\mathfrak{F}=\mathfrak{F}_{1}$.

Возьмем произвольную групшу $G$ из $\mathfrak{F}$. Тогда $G^{\mathfrak{H}} \in \mathfrak{M}$. Если $A \in K\left(G^{\mathfrak{H}}\right)$, то $A \in$ $K(\mathfrak{M})$. Из свойств радикала и корадикала получим $\left(G / G_{\beta(A)}\right)^{\mathfrak{H}} \cong G^{\mathfrak{H}} /\left(G_{\beta(A)} \cap G^{\mathfrak{H}}\right)=$ $G^{\mathfrak{H}} /\left(G^{\mathfrak{H}}\right)_{\beta(A)} \in m(A)$. Отсюда $G / G_{\beta(A)} \in m(A) \mathfrak{H}=f(A)$ для любого $A \in K\left(G^{\mathfrak{H}}\right)$. Пусть $A \in K(G) \backslash K\left(G^{\mathfrak{H}}\right)$. Таким образом, $G^{\mathfrak{H}} \subseteq O_{A^{\prime}}(G) \subseteq G_{\alpha(A)} \subseteq G_{\beta(A)}$. Тогда из того, что $G / G^{\mathfrak{H}} \in \mathfrak{H}$, имеем $G / G_{\beta(A)} \in \mathfrak{H} \subseteq f(A)$ для любого $A \in K(G) \backslash K\left(G^{\mathfrak{H}}\right)$. Следовательно, $G \in \mathfrak{F}_{1}$ и $\mathfrak{F} \subseteq \mathfrak{F}_{1}$.

Пусть $G \in \mathfrak{F}_{1} \backslash \mathfrak{F}$ и $G$ - группа минимального порядка с таким свойством. Тогда $G-$ монолитическая с монолитом $R=G^{\mathfrak{F}}$.

Из того, что $\mathfrak{H} \subseteq \mathfrak{F}$ и $G \notin \mathfrak{F}$, получаем $G \notin \mathfrak{H}$. Следовательно, $G^{\mathfrak{H}} \neq 1$ и $R \subseteq G^{\mathfrak{H}}$. Поскольку $G / R \in \mathfrak{F}=\mathfrak{M} \mathfrak{H}$, то $(G / R)^{\mathfrak{H}} \cong G^{\mathfrak{H}} /\left(R \cap G^{\mathfrak{H}}\right)=G^{\mathfrak{H}} / R \in \mathfrak{M}$.

Пусть $K(R)=(A)$. Так как $G \in \mathfrak{F}_{1}$, то $G / G_{\beta(A)} \in f(A) \neq \varnothing$. Если $A \in K(\mathfrak{M})$, то $G / G_{\beta(A)} \in m(A) \mathfrak{H}$, т.е. $\left(G / G_{\beta(A)}\right)^{\mathfrak{H}} \cong G^{\mathfrak{H}} /\left(G^{\mathfrak{H}}\right)_{\beta(A)} \in m(A)$. Следовательно, по лемме $2[4] G^{\mathfrak{H}} \in \mathfrak{M}$ и $G \in \mathfrak{M H}=\mathfrak{F}$. Противоречие. Пусть $A \in K(\mathfrak{H}) \backslash K(\mathfrak{M})$. Из условия леммы следует, что $A$ - простая неабелева группа. Тогда $G / G_{\beta(A)}=G / O_{A^{\prime}}(G) \cong G \in$ $f(A)=\mathfrak{H} \subseteq \mathfrak{F}$. Противоречие. Значит, $\mathfrak{F}_{1} \subseteq \mathfrak{F}$. Таким образом, $\mathfrak{F}=\mathfrak{F}_{1}$. Лемма доказана.

ЛЕмма 8. Пусть формация $\mathfrak{F}$ содержит лишь конечное число попарно неизоморфных формационно критических групп. Тогда если $G^{\mathfrak{F}}$ не имеет фраттиниевых $G$-главных факторов, то в формачии $\mathfrak{M}=F_{n}(G, \beta)$ имеется лишь конечное число п-кратно расслоенных подформачий с направлением $\beta$.

ДокАЗАТЕЛЬСТво. Проведем индукцию по $n$. Пусть $n=0$. Тогда $\mathfrak{M}=$ form $G$. По теореме $3.44[6] \mathfrak{M}$ имеет лишь конечное множество подформаций.

Пусть теперь $n>0$ и лемма верна для $(n-1)$. Обозначим через $m$ минимальный спутник формации $\mathfrak{M}$. По лемме $4 m(B)=F_{n-1}\left(G / G_{\beta(B)}, \beta\right)$ для любого $B \in$ $K(G)$ и $m(B)=\varnothing$, если $B \in \mathfrak{I} \backslash K(G)$. Ввиду теоремы 3.36 [6] $\mathfrak{F}$-корадикал группы $G / G_{\beta(B)}$ не содержит фраттиниевых $G$-главных факторов. Значит, по индукции в 
формации $F_{n-1}\left(G / G_{\beta(B)}, \beta\right)$ имеется лишь конечное множество $(n-1)$-кратно расслоенных подформаций с направлением $\beta$. Так как множество неизоморфных групп, принадлежаших $K(G)$, конечно, существует лишь конечное число спутников $t n$-кратно расслоенных формаций с условием $t \leqslant m$. Применяя теперь следствие 1 , заключаем, что в формации $\mathfrak{M}$ содержится лишш конечное множество $n$-кратно расслоенных подформаций с направлением $\beta$. Лемма доказана.

Из следствия 3.48 [6] и леммы 8 вытекает

ЛЕмма 9. Если S-корадикал $G^{\mathfrak{S}}$ группь $G$ не имеет фраттиниевых $G$-главных факторов, то в формачии $F_{n}(G, \beta)$ имеется лишь конечное мнохсество $n$-кратно расслоенных подформачий с направлением $\beta$.

Лемма 10. Пусть $\mathfrak{F} u \mathfrak{H}-\oint о р м а и и и ~ и \mathfrak{F} \nsubseteq \mathfrak{H}$, причем, по крайней мере одна из формачий $\mathfrak{F}$ и $\mathfrak{H}$ разрешима. Если $\mathfrak{F}-n$-кратно расслоенная формачия $c$ br-направлением $\beta, \alpha \leqslant \beta \leqslant \gamma$, то она обладает минимальной $n$-кратно расслоенной не $\mathfrak{H}$-подформачией с направлением $\beta$.

ДокАЗАТЕЛЬСтво. Утверждение очевидно при $\mathfrak{H}=\varnothing$. Пусть $\mathfrak{H} \neq \varnothing$ и $G$ - группа минимального порядка из $\mathfrak{F} \backslash \mathfrak{H}$. Тогда $G$ - монолитическая группа с монолитом $R=$ $G^{\mathfrak{H}}$. Индукцией по $n$ покажем, что в $\mathfrak{M}=F_{n}(G, \beta)$ имеется лишь конечное множество $n$-кратно расслоенных подформаций с направлением $\beta$.

Пусть $n=0$. Тогда $\mathfrak{M}=$ form $G$. По лемме $19.6[6] \mathfrak{M}$ имеет лишь конечное множество подформаций.

Пусть $n>0$ и лемма верна для $(n-1)$. Используя лемму 9 и методы леммы 8 , можно показать, что в формации $\mathfrak{M}$ содержится лишь конечное множество $n$-кратно расслоенных подформаций с направлением $\beta$. Так как при этом $\mathfrak{M} \nsubseteq \mathfrak{H}$, но $(1) \subseteq \mathfrak{H}$, то в $\mathfrak{M}$ можно выбрать такую $n$-кратно расслоенную подформацию $\mathfrak{K}$ с направлением $\beta$, что $\mathfrak{K} \nsubseteq \mathfrak{H}$. Но $\mathfrak{K}_{1} \subseteq \mathfrak{H}$ для каждой собственной $n$-кратно расслоенной подформации $\mathfrak{K}_{1} \mathrm{c}$ направлением $\beta$ из $\mathfrak{K}$. Лемма доказана.

Лемма 11 (М. М. Сорокина). Пусть $\mathfrak{H}$ - непустая расслоенная формация $c$ br-на-

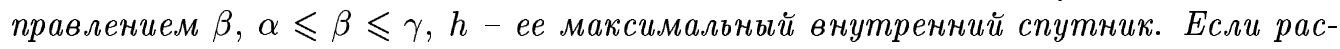
слоенная формация $\mathfrak{F}$ с направлением $\beta$ является минимальной расслоенной не $\mathfrak{H}$-формачией, то $\mathfrak{F}=F(G, \beta)$, где $G$ - такая монолитическая әруппа с монолитом $P=G^{\mathfrak{H}}$, что выполняется одно из следующих условий:

1) $G=P-$ группа простого порядка;

2) $P$ - неабелева группа $и P=G^{h(A)}$;

3) $G=[P] H$, где $P=C_{G}(P)$ - p-группа и $H=[Q] N-$ монолитическая группа с монолитом $Q=H^{h(A)}$ для $A \in K(P)$.

Лемма 12. Пусть $\mathfrak{F}$ - минимальная расслоенная не $\mathfrak{N}^{n}$-формация с br-направлением $\beta, \alpha \leqslant \beta \leqslant \gamma, n>1$. Тогда $\mathfrak{F}=F(G, \beta)$, әде $G$ - такая монолитическая группа с монолитом $P=G^{\mathfrak{N}^{n}}$, что выполняется одно из следующих условий:

1) $P$ - неабелева группа;

2) $G=[P] H$, где $P=C_{G}(P)$ - p-гpynпа и $H=[Q] N$, причем $C_{H}(Q)=Q=H^{\mathfrak{N}^{n-1}}$. 
ДокАЗАТЕЛЬСТВо. По лемме 2 и лемме 7 формация $\mathfrak{N}^{n}$ имеет такой максимальньй внутренний спутник $h$, что $h\left(Z_{p}\right)=\mathfrak{N}_{p} \mathfrak{N}^{n-1}$ для всех $Z_{p}, p \in \mathbb{P}$, и $h(A)=\mathfrak{N}^{n}$ для любой простой неабелевой групшы $A$. Ввиду леммы 11 имеем, что $\mathfrak{F}=F(G, \beta)$, где $G$ - такая монолитическая групша с монолитом $P=G^{\mathfrak{N}^{n}}$, что вьполняется одно из следуюших условий:

(a) $P$ - неабелева группа и $P=G^{h(A)}$ для $A \in K(P)$;

(b) $G=[P] H$, где $P=C_{G}(P)-p$-группа, а $H \neq 1$ - монолитическая группа с монолитом $Q=H^{h(A)}$ для $A \in K(P)$.

Пусть верно (b). Так как $G / P \cong H \in \mathfrak{N}^{n}$, то $Q$ абелева. Поскольку $H \notin h\left(Z_{p}\right)=$ $\mathfrak{N}_{p} \mathfrak{N}^{n-1}$, то $H \notin \mathfrak{N}$. Но формация $\mathfrak{N}_{p} \mathfrak{N}^{n-1}$ локальна, а значит, насьшена. Из того, что $H / Q \in \mathfrak{N}_{p} \mathfrak{N}^{n-1}$, заключаем, что $\Phi(H)=1$; тогда $H=[Q] N$. Из монолитичности $H$ следует, что $Q=C_{H}(Q)=F(H)$. Таким образом, $Q=H^{\mathfrak{N}^{n-1}}$. Лемма доказана.

ЛЕмма 13. Пусть $h-$ произвольный спутник расслоенной формации $\mathfrak{F}$ с br-направлением $\beta, \alpha \leqslant \beta \leqslant \gamma$. Тогда для минимального спутника $f$ имеет место: $f(A)=\operatorname{form}\left(G: G \in h(A) \cap \mathfrak{F} u O_{B}(G)=1\right.$ для всех $\left.B \in K(\mathfrak{F}) \backslash(A)\right)$, если $A-$ неабелева зруппа, и $f(A)=\operatorname{form}\left(G: G \in h(A) \cap \mathfrak{F} u O_{A}(G)=1\right)$ для абелевой zpynnы $A$.

ДокАЗАТЕЛЬСТво. По теореме $5[5] f(A)=\operatorname{form}\left(G / G_{\beta(A)} \mid G \in \mathfrak{F}\right)$ для любого $A \in K(\mathfrak{F})$ и $f(A)=\varnothing$, если $A \in \mathfrak{I} \backslash K(\mathfrak{F})$. Пусть $A \in K(\mathfrak{F}) \backslash \mathfrak{A}_{\text {и }} \mathfrak{F}_{A}=(G: G \in h(A) \cap$ $\mathfrak{F}$ и $O_{B}(G)=1$ для всех $\left.B \in K(\mathfrak{F}) \backslash(A)\right)$. Если $G \in \mathfrak{F}_{A}$, то $O_{A^{\prime}}(G)=G_{\beta(A)}=$ 1 и $G / G_{\beta(A)} \cong G \in f(A)$. Таким образом, $\mathfrak{F}_{A} \subseteq f(A)$. Для любой группы $S$ из $\mathfrak{F}$ выполняется $S / S_{\beta(A)} \in h(A) \cap \mathfrak{F}$. Но $O_{B}\left(S / S_{\beta(A)}\right)=O_{B}\left(S / O_{A^{\prime}}(S)\right)=1$ для всех $B \in$ $K(\mathfrak{F}) \backslash(A)$. Тогда $S / S_{\beta(A)} \in \mathfrak{F}_{A}$ и $f(A) \subseteq$ form $\mathfrak{F}_{A}$. Следовательно, $f(A)=$ form $\mathfrak{F}_{A}$ для любой неабелевой группы $A$ из $K(\mathfrak{F})$.

Возьмем произвольную группу $A$ из $K(\mathfrak{F}) \cap \mathfrak{A}$. Через $\mathfrak{F}_{A}$ теперь обозначим множество $\left(G: G \in h(A) \cap \mathfrak{F}\right.$ и $\left.O_{A}(G)=1\right)$. Для произвольной группы $M$ из $\mathfrak{F}_{A}$ рассмотрим регулярное сплетение $\Gamma=A$ 々 $M=[K] M$, где $K$ - база сплетения $\Gamma$. Несложно показать, что $O_{A^{\prime}, A}(\Gamma)=\Gamma_{\alpha(A)}=K$. Кроме того, по лемме $2[8] \Gamma_{\gamma(A)}=F_{A}(\Gamma)=K$. Тогда $\Gamma / \Gamma_{\beta(A)}=\Gamma / K \cong M \in h(A)$ и, по лемме $1 \Gamma \in \mathfrak{G}_{A} h(A) \subseteq \mathfrak{F}$. Но тогда $\Gamma / \Gamma_{\beta(A)} \cong M \in f(A)$ и, значит, $\mathfrak{F}_{A} \subseteq f(A)$. С другой стороны, для любой группы $N$ из $\mathfrak{F}$ вьполняется $N / N_{\beta(A)} \in h(A) \cap \mathfrak{F}$. Поскольку $\beta$ является $b$-направлением, по лемме 6 [4] $O_{A}\left(N / N_{\beta(A)}\right)=1$. Таким образом, $N / N_{\beta(A)} \in \mathfrak{F}_{A}$ и $f(A) \subseteq$ form $\mathfrak{F}_{A}$. Значит, $f(A)=$ form $\mathfrak{F}_{A}$ для любой абелевой группы $A$ из $K(\mathfrak{F})$. Лемма доказана.

Будем обозначать через $\triangle(\mathfrak{F})$ - множество всех попарно неизоморфных групп из $K(\mathfrak{F})$.

Лемма 14. Тогда и только тогда расслоенная формачия $\mathfrak{F}$ с br-направлением $\beta$, $\alpha \leqslant \beta \leqslant \gamma$, является однопорожсенной формачией, когда $\triangle(\mathfrak{F})$ - конечное множество и $\mathfrak{F}$ обладает таким внутренним спутником $f$, что $f(A)$ однопорохсдена для всех $A \in \mathfrak{I}$.

ДокАЗАТЕЛЬСТво. Необходимость. По условию $\mathfrak{F}=F(G, \beta)$ для некоторой группы $G$. По следствию $3 K(\mathfrak{F})=K(G)$. Кроме того, ввиду теоремы $5[5]$ формация $\mathfrak{F}$ имеет минимальный спутник $f$ такой, что все его значения будут однопорожденными формациями. 
Достаточность. Пусть $\triangle(\mathfrak{F})=\left\{A_{1}, A_{2}, \ldots, A_{t}\right\}$ и существует внутренний спутник $f$ такой, что $f(A)$ - однопорожденная формация для всех $A \in \mathfrak{I}$. В частности, $f\left(A_{i}\right)=$ $\operatorname{form}\left(G_{i}\right), i \in 1,2, \ldots, t$. Без ограничения общности можем считать, что $A_{1}, A_{2}, \ldots, A_{n}-$ абелевы группы, $n \leqslant t$, т.е. $A_{i} \cong Z_{p_{i}}$ для некоторого $p_{i} \in \mathbb{P}$, а $A_{n+1}, \ldots, A_{t}$ - простые неабелевы групшы. Для всякого $i \in 1,2, \ldots, n$ рассмотрим регулярное сплетение $B_{i}=$ $A_{i} \curlywedge\left(G_{i} / O_{A_{i}}\left(G_{i}\right)\right)=\left[K_{i}\right]\left(G_{i} / O_{A_{i}}\left(G_{i}\right)\right)$, где $K_{i}$ - база сплетения. Пусть $G=B_{1} \times \cdots \times$ $B_{n} \times G_{n+1} \times \cdots \times G_{t}$ и $\mathfrak{M}=F(G, \beta)$. Обозначим через $m$ - максимальньй внутренний спутник формации $\mathfrak{M}$ и покажем, что $\mathfrak{F}=\mathfrak{M}$.

Поскольку $f$ - внутренний спутник формации $\mathfrak{F}$, то $G_{i} \in \mathfrak{F}$. Более того, $B_{i} / K_{i} \cong$ $G_{i} / O_{A_{i}}\left(G_{i}\right) \in f\left(A_{i}\right)$ и по лемме $1 B_{i} \in \mathfrak{G}_{A_{i}} f\left(A_{i}\right) \subseteq \mathfrak{F}$ для всех $i \in 1,2, \ldots, n$. Таким образом, $\mathfrak{M} \subseteq \mathfrak{F}$.

По лемме $2 f\left(A_{i}\right) \subseteq m\left(A_{i}\right)=\mathfrak{M}$ для всех $i \in n+1, \ldots, t$. Кроме того, для $i \in 1,2, \ldots$ $\ldots, n$ вьполняется $B_{i} /\left(B_{i}\right)_{\beta\left(A_{i}\right)} \in m\left(A_{i}\right)$. Заметим, что для $i \in 1,2, \ldots, n\left(B_{i}\right)_{\beta\left(A_{i}\right)}=$ $K_{i}$. Тогда по лемме $2 \quad B_{i} \in \mathfrak{G}_{A_{i}} m\left(A_{i}\right)=m\left(A_{i}\right)$ и, следовательно, $B_{i} / K_{i} \cong$ $G_{i} / O_{A_{i}}\left(G_{i}\right) \in m\left(A_{i}\right)$ для всех $i \in 1,2, \ldots, n$. Таким образом, $G_{i} \in \mathfrak{G}_{A_{i}} m\left(A_{i}\right)=$ $m\left(A_{i}\right)$ для $i \in 1,2, \ldots, n$. В итоге получили, что $f \leqslant m$ и $\mathfrak{F} \subseteq \mathfrak{M}$. Лемма доказана.

ЛЕмма 15. Пусть $\mathfrak{F}=\mathfrak{M H}$ - несократимая факторизачия однопорожденной расслоенной формации $\mathfrak{F}$ с br-направлением $\beta, \alpha \leqslant \beta \leqslant \gamma$, где $\mathfrak{M}$ - такэсе расслоенная формаиия с направлением $\beta$. Тогда $\mathfrak{M}$ - метанильпотентная расслоенная формачия.

ДокАЗАТЕЛЬСТВо. По условию для некоторой групшы $G$ справедливо $\mathfrak{F}=F(G, \beta)$. Обозначим через $f$ - минимальный спутник формации $\mathfrak{F}$.

Покажем сначала, что $|\triangle(K(\mathfrak{M}) \cap \mathfrak{A})|>1$. Допустим, что существует простая неабелева группа $T \in \mathfrak{M}$. Для произвольной группы $H$ из $\mathfrak{H}$ рассмотрим регулярное сплетение $W=T \imath\left(H^{n}\right)=[K]\left(H^{n}\right)$, где $K$ - база сплетения $W$, а $H^{n}$ - прямое произведение $n$ экземпляров групшы $H$. По предложению 18.5 [8] группа $W$ монолитична с монолитом $R=K$. Несложно показать, что $W \in \mathfrak{F}$. Тогда по теореме 5 [5] $W / W_{\beta(T)} \cong W / O_{T^{\prime}}(W) \cong W \in f(T)=\operatorname{form}\left(G / G_{\beta(T)}\right)$. Ho $|R|=|K|=|T|^{|H|^{n}}$, что противоречит лемме 3.1 .5 [3]. Следовательно, все простые групшы в формации $\mathfrak{M}$ абелевы и $K(\mathfrak{M}) \cap \mathfrak{A} \neq \varnothing$.

Допустим, что $K(\mathfrak{M}) \cap \mathfrak{A}=\left(Z_{p}\right)$. Возьмем групу $Y$ из разности $\mathfrak{F} \backslash \mathfrak{H}$, причем минимальную с таким свойством. Тогда $Y$-монолитическая группа с монолитом $R=$ $Y^{\mathfrak{H}} \in \mathfrak{M}$. Как показано вьше, каждая простая группа из $\mathfrak{M}$ абелева. Значит, $R$ - элементарная абелева $p$-группа.

Допустим, что существует $U \in K(\mathfrak{M}) \backslash \mathfrak{A}$. Тогда существует группа $S \in \mathfrak{M}$ такая, что $U \in K(S)$. Без ограничения обшности можем считать, что $O_{U^{\prime}}(S)=1$. Для произвольного натурального числа $m$ рассмотрим регулярное сплетение $V=S \imath\left(Y^{m} / R^{m}\right)$. Допустим, что $V \in \mathfrak{F}$. Тогда $V / V_{\beta(U)} \cong V / O_{U^{\prime}}(V) \cong V \in f(U)=\operatorname{form}\left(G / O_{U^{\prime}}(G)\right)$, для любого $m \in \mathbb{N}$. Но это противоречит лемме 3.1 .5 [3]. Значит, $V \notin \mathfrak{F}$. Поскольку $V / K \in \mathfrak{H}$, где $K$ - база сплетения $V$, то $V^{\mathfrak{H}} \subseteq K \in \mathfrak{M}$. Но $V^{\mathfrak{H}}$ не входит подпрямо в $K$. Обозначим через $S_{1}$ - первую копию $S$ в $K$ и $V_{1}$ - проекцию $V^{\mathfrak{H}}$ на $S_{1}$. Поскольку $V^{\mathfrak{H}}$ нормальна в $V$, то $V_{1}$ нормальна в $S_{1}$. Так как $V^{\mathfrak{H}}$ не входит подпрямо в $K$, то $V_{1} \neq S_{1}$ и по лемме 3.1.9 [3] $\left(S_{1} / V_{1}\right)$ 乙 $\left(Y^{m} / R^{m}\right)$ - гомоморфньй образ $V / V^{\mathfrak{H}}$. Таким образом, учитывая лемму $18.2(\mathrm{~d})[8],\left(S_{1} / V_{1}\right) \prec\left(Y^{m} / R^{m}\right) \cong V /\left(V_{1}\right)^{\#} \in \mathfrak{H}$. Пусть $S_{1} / X_{1}-$ верхний 
фактор главного ряда $S_{1}$, проходящего через $V_{1}$. Тогда, $\left(X_{1}\right)^{\#}$ - нормальная подгруппа в $V$ и $\left(V_{1}\right)^{\#} \subseteq\left(X_{1}\right)^{\#}$. Значит, $V /\left(X_{1}\right)^{\#} \in \mathfrak{H}$. Поскольку $S_{1} / X_{1} \in \mathfrak{M}$ и $S_{1} / X_{1}-$ простая группа, то $S_{1} / X_{1} \cong Z_{p}$ и $V /\left(X_{1}\right)^{\#} \cong Z_{p} \imath\left(Y^{m} / R^{m}\right) \in \mathfrak{H}$. Но в этом случае по лемме 3.5.20 [3] $Y^{m} \in \operatorname{form}\left(Z_{p} \imath\left(Y^{m} / R^{m}\right)\right) \subseteq \mathfrak{H}$ и $Y \in \mathfrak{H}$. Получили противоречие с выбором $Y$. Значит, $K(\mathfrak{M}) \subseteq \mathfrak{A}$. По допушению $K(\mathfrak{M}) \cap \mathfrak{A}=\left(Z_{p}\right)$. Тогда $K(\mathfrak{M})=\left(Z_{p}\right)$ и $\mathfrak{M}=\mathfrak{N}_{p} \subseteq \mathfrak{N}^{2}$. Противоречие. Следовательно, $|\triangle(K(\mathfrak{M}) \cap \mathfrak{A})|>1$.

Приступим теперь непосредственно к доказательству метанильпотентности $\mathfrak{M}$. Предположим, что $\mathfrak{M} \nsubseteq \mathfrak{N}^{2}$. Тогда по лемме 10 в $\mathfrak{M}$ имеется неметанильпотентная расслоенная подформация $\mathfrak{M}_{0}$ с направлением $\beta$, у которой все собственные расслоенные подформации с направлением $\beta$ метанильпотентны. Причем, по лемме $12 \mathfrak{M}_{0}=F(Z, \beta)$, где группа $Z$ удовлетворяет одному из следующих условий:

(a) $P=Z^{\mathfrak{N}^{2}}$ - неабелева единственная минимальная нормальная подгруппа в $Z$;

(b) $Z=[P] H$, где $P=C_{Z}(P)-p$-групша, а $H=[Q] N \neq 1$, причем $Q=C_{H}(Q)=H^{\mathfrak{N}}$ - монолит группы $H$. Докажем, что оба эти случая невозможны.

Обозначим через $\mathfrak{F}_{1}$ формацию form $\left(G / G_{\beta(B)}\right.$, где $\left.B \in K(G)\right)$. Тогда $\mathfrak{F}_{1}=$ form $(\Gamma)$ для некоторой групш $\Gamma$. Пусть $A_{1}-$ произвольная монолитическая группа из $\mathfrak{H}$. Понятно, что $O_{Z_{p}}\left(A_{1}\right)=1$ для некоторого $Z_{p} \in K(\mathfrak{M})$. Значит, $A_{1} \in \mathfrak{H} \subseteq m\left(Z_{p}\right) \mathfrak{H}$ и по лемме $13 A_{1} \in f\left(Z_{p}\right)=\operatorname{form}\left(G / G_{\beta\left(Z_{p}\right)}\right) \subseteq \mathfrak{F}_{1}$. Следовательно, всякая монолитическая группа из $\mathfrak{H}$ входит в $\mathfrak{F}_{1}$. Поэтому $\mathfrak{H} \subseteq \mathfrak{F}_{1}$.

Пусть $M-$ неединичная группа из $\mathfrak{H}$. Для всякого натурального числа $n$ через $G_{n}$ обозначим регулярное сплетение $G_{n}=Z \imath\left(M^{n}\right)=[K]\left(M^{n}\right)$, где $K$ - база сплетения. Так как $G_{n} / K \cong M^{n} \in \mathfrak{H}$, то $\left(G_{n}\right)^{\mathfrak{H}} \subseteq K \in \mathfrak{M}$. Пусть $D_{1}-$ проекция $\left(G_{n}\right)^{\mathfrak{H}}$ в первую копию $Z_{1}$. Допустим, что $D_{1} \neq Z_{1}$. Поскольку $\left(G_{n}\right)^{\mathfrak{H}}$ нормальна в $G_{n}$, то $D_{1}$ нормальна в $Z_{1}$. По лемме 3.1 .9 [3] $\left(Z_{1} / D_{1}\right)$ 乙 $\left(M^{n}\right)$ - гомоморфньй образ групшы $G_{n} /\left(G_{n}\right)^{\mathfrak{H}}$, т.е. $\left(Z_{1} / D_{1}\right)$ ᄂ $\left(M^{n}\right) \in \mathfrak{H}$. Следовательно, $\pi\left(Z_{1} / D_{1}\right) \cap \pi(\mathfrak{H}) \neq \varnothing$. Тогда без ограничения общности в качестве $M$ можно взять прямое произведение $q d$-групп из $\mathfrak{H}$, где $q \in \pi\left(Z_{1} / D_{1}\right) \cap \pi(\mathfrak{H})$, по одной для каждого $q$. Если $L-$ подгруппа порядка $q$ из $M$, a $T$ - подгруппа порядка $q$ из $Z_{1} / D_{1}$, то по лемме 18.2 [8] групша $T$ ? $\left(L^{n}\right)$ изоморфно вкла-

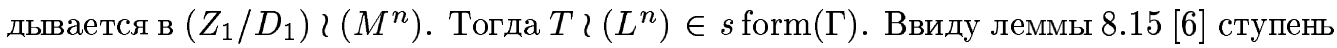
нильпотентности группы $T$ ? $\left(L^{n}\right)$ не меньше $n+1$. Противоречие с леммой 3.1 .5 [3]. Таким образом, $Z_{1}=D_{1}$, т.е. $\left(G_{n}\right)^{\mathfrak{H}}$ входит подпрямо в $K$. Поэтому $\left(G_{n}\right)^{\mathfrak{H}} \in \mathfrak{M}$ и $G_{n} \in \mathfrak{M H}=\mathfrak{F}$.

Пусть $Z$ удовлетворяет условию (а), $B \in K(P)$. Поскольку каждая простая группа в $\mathfrak{M}$ абелева, то $P \subset Z$. Тогда $G_{n} / O_{B^{\prime}}\left(G_{n}\right) \cong G_{n} \in f(B)=\operatorname{form}\left(G / O_{B^{\prime}}(G)\right)$. Ho $G_{n}$ содержит монолит порядка $|P|^{|M|^{n}}$, что противоречит лемме 3.1.5 [3].

Пусть $Z$ удовлетворяет условию (b), $p \in \pi(P)$. Так как $C_{Z}(P)=P$, то $P$ не содержится в центре $Z$. Следовательно, по предложению 18.5 [8] $G_{n}$ - монолитична с монолитом $T=\prod_{b \in M^{n}} P_{1}^{b}$, где $P_{1}-$ монолит $Z_{1}$. Из локальности $\mathfrak{N}^{2}$ заключаем, что $P \nsubseteq \Phi(Z)$. Тогда ввиду леммы $9.4[8] T \nsubseteq \Phi(K)$ и $T$ - дополняема в $G_{n}$. Из монолитичности $G_{n}$ заключаем, что $C_{G_{n}}(T)=T$. Таким образом, $\left(G_{n}\right)_{\beta\left(Z_{p}\right)}=T$. Поэтому $W=G_{n} / T \cong\left(Z_{1} / P_{1}\right) \imath\left(M^{n}\right) \in f\left(Z_{p}\right)$. Но $Z_{1} / P_{1} \cong H$ и $C_{H}(Q)=Q$. Значит, $Q$ не содержится в центре $H$ и по предложению 18.5 [8] $W$ имеет минимальную нормальную подгрупшу порядка $|Q|^{|M|^{n}}$. Это приводит к противоречию с леммой 3.1 .5 [3].

Следовательно, $\mathfrak{M} \subseteq \mathfrak{N}^{2}$. Лемма доказана.

Через $\mathfrak{H} / O_{p}(\mathfrak{H})$ будем обозначать формацию, порожденную всеми группами 
$G / O_{p}(G)$, где $G \in \mathfrak{H}$.

ТЕОРема 1. Пусть $\mathfrak{F}=\mathfrak{M H}-$ несократимая факторизация расслоенной формаиии $\mathfrak{F}$ с br-направлением $\beta, \alpha \leqslant \beta \leqslant \gamma$, где $\mathfrak{M}$ - также расслоенная формачия $c$ направлением $\beta$. Тогда и только тогда $\mathfrak{F}$ - однопорохсденная расслоенная формачия, когда выполняются следующие условия:

1) $K(\mathfrak{H}) \cap \mathfrak{A} \subseteq K(\mathfrak{M})$;

2) $\mathfrak{M}$ - метанильпотентная однопорожденная расслоенная формация;

3) $\pi(\mathfrak{H}) \cap \pi(m(B))=\varnothing$ для всех $B \in K(\mathfrak{M})$, где $m$ - минимальный спутник формаиии $\mathfrak{M}$

4) если $|\pi(\mathfrak{M})|>1$, то $\mathfrak{H}-$ однопорожденная формаиия, причем $\mathfrak{M} \nsubseteq \mathfrak{N}$ влечет $\mathfrak{H} \subseteq \mathfrak{A}$

5) если $\mathfrak{M}=\mathfrak{N}_{p}$, то формация $\mathfrak{H} / O_{p}(\mathfrak{H})$ однопорожсдена.

ДокАЗАТЕЛЬСТво. Необходимость. По условию для некоторой группы $G$ справедливо $\mathfrak{F}=F(G, \beta)$. Обозначим через $h-$ минимальный спутник формации $\mathfrak{F}$.

По лемме $15 \mathfrak{M}$ - метанильпотентная формация.

Допустим, что $K(\mathfrak{H}) \cap \mathfrak{A} \nsubseteq K(\mathfrak{M})$. Тогда по лемме 6 в формации $\mathfrak{F}$ содержится бесконечное множество наследственных расслоенных подформаций с направлением $\beta$, что противоречит лемме 5. Значит, $K(\mathfrak{H}) \cap \mathfrak{A} \subseteq K(\mathfrak{M})$, т.е. выполняется условие 1$)$.

Обозначим через $m$ минимальньй спутник формации $\mathfrak{M}$. По лемме 7 формация $\mathfrak{F}$ имеет также такой спутник $f$, что $f(B)=m(B) \mathfrak{H}$ для всех $B \in K(\mathfrak{M}), f(B)=\mathfrak{H}$ при всяком $B \in K(\mathfrak{H}) \backslash K(\mathfrak{M})$. Поскольку $m$ - внутренний спутник формации $\mathfrak{M}$, то $f$ - внутренний спутник формации $\mathfrak{F}$. Предположим, что найдутся такие различные простые числа $p$ и $q$, что в формации $m\left(Z_{p}\right)$ содержится группа $Z_{q}$ и $q \in \pi(\mathfrak{H})$. Пусть $H$ - некоторая $q d$-группа из $\mathfrak{H}$. Для всякого натурального числа $n$ через $H_{n}$ обозначим регулярное сплетение $Z_{q}$ २ $\left(H^{n}\right)=[K]\left(H^{n}\right)$, где $K$ - база сплетения. Так как $H_{n} / K \cong H^{n} \in \mathfrak{H}$, т.е. $\left(H_{n}\right)^{\mathfrak{H}} \subseteq K \in m\left(Z_{p}\right)$, то $H_{n} \in m\left(Z_{p}\right) \mathfrak{H}$. По лемме 18.8(в) [8] $O_{p}\left(H_{n}\right)=1$. Следовательно, по лемме $13 H_{n} \in h\left(Z_{p}\right)$. Если $L$ - подгруппа порядка $q$ из $H$, то по лемме 18.2 [8] группа $Z_{q}$ ? $\left(L^{n}\right)$ изоморфно вкладывается в $H_{n}$. Ввиду леммы 8.15 [6] ступень нильпотентности группы $Z_{q}$ ? $\left(L^{n}\right)$ не меньше $n+1$. По теореме 5 [5] $h\left(Z_{p}\right)=\operatorname{form}\left(G / G_{\beta\left(Z_{p}\right)}\right)$. Обозначим через $\mathfrak{H}_{1}-$ формацию, порожденную всеми силовскими $q$-подгрупшами из $\left.G / G_{\beta(} Z_{p}\right)$. По лемме 8.11 [6] всякая силовская $q$-подгрупша группы $H_{n}$ принадлежит $\mathfrak{H}_{1}$. Хорошо известно, что формация, порожденная конечным множеством групп, однопорождена; пусть $\mathfrak{H}_{1}=$ form $S$ для некоторой группы $S$. Таким образом, $Z_{q}$ ᄀ $\left(L^{n}\right) \in s$ form $S$. Последнее ввиду леммы 3.1.5 [3] означает, что ступень нильпотентности группы $Z_{q}$ ? $\left(L^{n}\right)$ не превьшает (при любом натуральном $n$ ) ступень нильпотентности группы $S$. Противоречие.

Итак, в дальнейшем мы можем считать, что если $p$ и $q-$ различные простые числа и группа $Z_{q}$ принадлежит формации $m\left(Z_{p}\right)$, то $q \notin \pi(\mathfrak{H})$. Более того, как и при доказательстве теоремы 8.16 [6], можно показать, что в этой ситуации формация $\mathfrak{H}$ абелева.

Покажем, что $\mathfrak{M}$ - метанильпотентная однопорожденная расслоенная формация. Ввиду следствия 4.1 [5] и леммы 8.10 [6] всякая расслоенная подформация с направлением $\beta$ из $\mathfrak{M}$ наследственна. Следовательно, $\mathfrak{M} \subseteq \mathfrak{F}$ и по лемме 5 в $\mathfrak{M}$ имеется лишш конечное множество расслоенных подформаций с направлением $\beta$. Пусть $(1)=\mathfrak{M}_{0} \subset \mathfrak{M}_{1} \subset \cdots$ $\subset \mathfrak{M}_{k}=\mathfrak{M}-$ такая цеп расслоенных формаций с направлением $\beta$, что $\mathfrak{M}_{i-1}-$ мак- 
симальная расслоенная подформация в $\mathfrak{M}_{i}, i=1,2, \ldots, k$. Пусть $H_{i} \in \mathfrak{M}_{i} \backslash \mathfrak{M}_{i-1}$, $i=1,2, \ldots, k$. Тогда $\mathfrak{M}=F\left(H_{1}, \ldots, H_{k}, \beta\right)=F\left(H_{1} \times \cdots \times H_{k}, \beta\right)$, т.е. $\mathfrak{M}$ - однопорожденная расслоенная формация. Итак, вьполняется условие 2$)$.

Предположим, что найдется такое простое число $q$, что $q \in \pi(\mathfrak{H}) \cap \pi(m(B))$. Так как формация $\mathfrak{M}$ метанильпотентна, то $K(\mathfrak{M}) \subseteq \mathfrak{A}$, т.е. $B \cong Z_{p}$ для некоторого $p \in \mathbb{P}$. Причем, по следствию $5.1[5] m\left(Z_{p}\right) \subseteq m_{1}\left(Z_{p}\right)$, где $m_{1}$ - минимальньй спутник формации $\mathfrak{N}^{2}$. По лемме $7 m\left(Z_{p}\right) \subseteq \mathfrak{N}$. Более того, учитьвая лемму 13 , заключаем, что $m\left(Z_{p}\right) \subseteq \mathfrak{N}_{p^{\prime}}$. Следовательно, $q \neq p$ и в формации $m\left(Z_{p}\right)$ имеется группа порядка $q$. Значит, как показано ранее, $q \notin \pi(\mathfrak{H})$. Противоречие. Таким образом, $\mathfrak{M}$ и $\mathfrak{H}$ удовлетворяют условию 3).

Пусть $\mathfrak{M} \nsubseteq \mathfrak{N}$. Тогда найдется такое $Z_{p} \in K(\mathfrak{M})$, что $(1) \subset m\left(Z_{p}\right)$. Hо $m\left(Z_{p}\right) \subseteq \mathfrak{N}_{p^{\prime}}$. Следовательно, в формации $m\left(Z_{p}\right)$ имеется группа простого порядка $q$, где $p \neq q$. В этом случае ввиду установленного ранее формация $\mathfrak{H}$ абелева.

Предположим теперь, что $|\pi(\mathfrak{M})|>1$. Напомним, что через $\mathfrak{F}_{1}$ мы обозначили формацию $\mathfrak{F}_{1}=\operatorname{form}\left(G / G_{\beta(B)}\right.$, где $\left.B \in K(G)\right)$. Пусть $\mathfrak{H} \subseteq \mathfrak{A}$. Рассуждая, как показано выше, можно показать, что $\mathfrak{H} \subseteq \mathfrak{F}_{1}$. Тогда по лемме 3.1 .5 [3] число неизоморфных монолитических групп из $\mathfrak{H}$ конечно, ибо все они являются циклическими группами порядка не больше $|G|$. Значит, в этом случае формация $\mathfrak{H}$ однопорождена. Пусть $\mathfrak{H} \nsubseteq \mathfrak{A}$. Тогда ввиду доказанного ранее $\mathfrak{M} \subseteq \mathfrak{N}$. Следовательно, $m\left(Z_{p}\right)=(1)$ при всех $Z_{p} \in K(\mathfrak{M})$. Таким образом, $f\left(Z_{p}\right)=m\left(Z_{p}\right) \mathfrak{H}=\mathfrak{H}$ и $f(B)=\mathfrak{H}$ для $B \in K(\mathfrak{H}) \backslash(\mathfrak{M})$. Но $h \leqslant f$. Значит, $\mathfrak{F}_{1} \subseteq \mathfrak{H}$. Получаем $\mathfrak{H}=\mathfrak{F}_{1}-$ однопорожденная формация.

Возьмем теперь $\mathfrak{M}=\mathfrak{N}_{p}$. Покажем, что в этом случае $\mathfrak{F}=\mathfrak{N}_{p} \mathfrak{H}=\mathfrak{N}_{p}\left(\mathfrak{H} / O_{p}(\mathfrak{H})\right)$. Очевидно, $\mathfrak{N}_{p}\left(\mathfrak{H} / O_{p}(\mathfrak{H})\right) \subseteq \mathfrak{N}_{p} \mathfrak{H}$. Пусть теперь $S \in \mathfrak{N}_{p} \mathfrak{H}$. Тогда $S^{\mathfrak{H}} \in \mathfrak{N}_{p}$, т.е. $S^{\mathfrak{H}} \subseteq$ $O_{p}(S)$. Значит, $S / O_{p}(S) \in \mathfrak{H}$. Но $O_{p}\left(S / O_{p}(S)\right)=1$ и, следовательно, $S / O_{p}(S) \in$ $\mathfrak{H} / O_{p}(\mathfrak{H})$. Поэтому $S \in \mathfrak{N}_{p}\left(\mathfrak{H} / O_{p}(\mathfrak{H})\right)$ и $\mathfrak{N}_{p} \mathfrak{H} \subseteq \mathfrak{N}_{p}\left(\mathfrak{H} / O_{p}(\mathfrak{H})\right)$. По лемме $7 \mathfrak{F}$ имеет спутник $f_{1}$ такой, что $f_{1}(A)=\mathfrak{H} / O_{p}(\mathfrak{H})$ для всех $A \in K(\mathfrak{F})$. Пусть $M-$ произвольная группа из $\mathfrak{H}$. Тогда $M / O_{p}(M) \in \mathfrak{H} / O_{p}(\mathfrak{H})=f_{1}\left(Z_{p}\right)$. Поскольку $O_{p}\left(M / O_{p}(M)\right)=1$, по лемме $13 M / O_{p}(M) \in h\left(Z_{p}\right) \subseteq \mathfrak{F}_{1}$. Следовательно, $\mathfrak{H} / O_{p}(\mathfrak{H}) \subseteq \mathfrak{F}_{1}$. По определению $h \leqslant f_{1}$. Тогда $\mathfrak{F}_{1} \subseteq \mathfrak{H} / O_{p}(\mathfrak{H})$ и $\mathfrak{F}_{1}=\mathfrak{H} / O_{p}(\mathfrak{H})$ - однопорожденная формация.

Достаточность. Пусть $\pi(\mathfrak{M})=\{p\}$. Ввиду условия 2$)$ заключаем, что $\mathfrak{N}_{p} \subseteq \mathfrak{M}$. Таким образом, $\mathfrak{N}_{p}=\mathfrak{M}_{\text {и }} \mathfrak{F}=\mathfrak{N}_{p} \mathfrak{H}$. Как говорилось вьше, $\mathfrak{F}=\mathfrak{N}_{p} \mathfrak{H}=\mathfrak{N}_{p}\left(\mathfrak{H} / O_{p}(\mathfrak{H})\right)$ и по лемме $7 \mathfrak{F}$ имеет спутник $f_{1}$ такой, что $f_{1}(C)=\mathfrak{H} / O_{p}(\mathfrak{H})$ для всех $C \in K(\mathfrak{F})$. Из теорем 1 и $2[5]$ следует, что $\mathfrak{H} / O_{p}(\mathfrak{H})$ является свободной формацией. Тогда, учитывая теорему $5[5]$, легко показать, что $\triangle\left(\mathfrak{H} / O_{p}(\mathfrak{H})\right)$ - конечное множество. Но в этом случае $\triangle(\mathfrak{F})$ - конечное множество. Следовательно, по лемме $14 \mathfrak{F}$ - однопорожденная формация.

Рассмотрим случай, когда $|\pi(\mathfrak{M})|>1$. Ввиду леммы 7 формация $\mathfrak{F}$ имеет такой спутник $f$, что $f(C)=m(C) \mathfrak{H}$, если $C \in K(\mathfrak{M})$, и $f(C)=\mathfrak{H}$ для всех $C \in K(\mathfrak{H}) \backslash K(\mathfrak{M})$. По условию 2) $\mathfrak{M}$ - однопорожденная расслоенная формация. Значит, по лемме $14 \triangle(\mathfrak{M})-$ конечное множество. По теореме 1 и теореме 2 [5] следует, что $\mathfrak{H}$ является свободной формацией. Тогда из теоремы $5[5]$ несложно получить, что $\triangle(\mathfrak{H})$ - конечное множество. Очевидно, что $K(\mathfrak{F}) \subseteq K(\mathfrak{H}) \cup K(\mathfrak{M})$. Поэтому $\triangle(\mathfrak{F})$ - конечное множество.

Используя рассуждения, приведенные в доказательстве теоремы 8.16 [6], несложно показать, что формация $m(C) \mathfrak{H}$ однопорождена для любого $C \in K(\mathfrak{M})$. Тогда по лемме $14 \mathfrak{F}$ является однопорожденной расслоенной формацией с направлением $\beta$. Теорема 
доказана.

ТЕорема 2. Пусть $\mathfrak{F}=\mathfrak{M}_{1} \ldots \mathfrak{M}_{t}-$ несократимая факторизация расслоенной формачии $\mathfrak{F}$ с br-направлением $\beta, \alpha \leqslant \beta \leqslant \gamma$, причем формачия $\mathfrak{M}_{1}$ также является расслоенной с направлением $\beta$. Формация $\mathfrak{F}$ тогда и только тогда однопорождена, когда выполняются следующие условия:

1) $t \leqslant 3, K(\mathfrak{F}) \cap \mathfrak{A}=K\left(\mathfrak{M}_{1}\right) u|\triangle(\mathfrak{F})|>1$;

2) при $t=2$ выполняются следующие пункты:

- $\mathfrak{M}_{1}$ - метанильпотентная однопорожсенная расслоенная формация;

- $\pi\left(\mathfrak{M}_{2}\right) \cap \pi(m(B))=\varnothing$ для всех $B \in K\left(\mathfrak{M}_{1}\right)$, әде $m-$ минимальный спутник формачии $\mathfrak{M}_{1}$

- если $\left|\pi\left(\mathfrak{M}_{1}\right)\right|>1$, то $\mathfrak{M}_{2}$ - однопорожденная формачия, причем $\mathfrak{M}_{1} \nsubseteq \mathfrak{N}$ влечет $\mathfrak{M}_{2} \subseteq \mathfrak{A}$;

- если $\left|\pi\left(\mathfrak{M}_{1}\right)\right|=1$, то $\mathfrak{M}_{2} / O_{p}\left(\mathfrak{M}_{2}\right)$ - однопорожденная формачия;

3) если $t=3$, то $\mathfrak{M}_{1}$ и $\mathfrak{M}_{1} \mathfrak{M}_{2}$ - однопорожсденне расслоенные формачии $c$ направлением $\beta, \mathfrak{M}_{2}, \mathfrak{M}_{3}, \mathfrak{M}_{2} \mathfrak{M}_{3}$ однопорождень, причем, формачии $\mathfrak{M}_{1}$, $\mathfrak{M}_{2}$ нильпотентны, формачия $\mathfrak{M}_{3}$ абелева и $\pi\left(\mathfrak{M}_{2}\right) \cap \pi\left(\mathfrak{M}_{3}\right)=\varnothing$.

ДокАЗАТЕЛЬСтво. Необходимость. Пусть $\mathfrak{H}_{1}=\mathfrak{M}_{2} \ldots \mathfrak{M}_{t}$, т.е. $\mathfrak{F}=\mathfrak{M}_{1} \mathfrak{H}_{1}$ и $\mathfrak{F}=$ $F(G, \beta)$ для некоторой групш $G$. По теореме $1 \mathfrak{M}_{1}$ - метанильпотентная расслоенная формация. Значит, существует $p \in \mathbb{P}$ для которого $\mathfrak{N}_{p} \subseteq \mathfrak{M}_{1}$. Очевидно, что $\mathfrak{N}_{p} \subseteq \mathfrak{F}$ для всех $p \in \pi\left(\mathfrak{M}_{1}\right)$. Предположение, что $\mathfrak{F}=\mathfrak{N}_{p}$, противоречит следствию 3.3 .7 [3]. Таким образом, $|\triangle(\mathfrak{F})|>1$. Покажем, что $K(\mathfrak{F}) \cap \mathfrak{A}=K\left(\mathfrak{M}_{1}\right)$. Для любого $Z_{p} \in K(\mathfrak{F})$ выполняется $\mathfrak{N}_{p}=\mathfrak{N}_{p} \cap \mathfrak{F}=\left(\mathfrak{N}_{p} \cap \mathfrak{M}_{1}\right)\left(\mathfrak{N}_{p} \cap \mathfrak{H}_{1}\right)$. По следствию 3.3 .7 [3] формации $\mathfrak{N}_{p} \cap \mathfrak{M}_{1}$ и $\mathfrak{N}_{p} \cap \mathfrak{H}_{1}$ не могут одновременно быть отличны от (1) и $\mathfrak{N}_{p}$. Поэтому либо $\mathfrak{N}_{p} \subseteq \mathfrak{M}_{1}$, либо $\mathfrak{N}_{p} \subseteq \mathfrak{H}_{1}$. Допустим, что найдется $\mathfrak{N}_{p} \subseteq \mathfrak{H}_{1}$ и $\mathfrak{N}_{p} \nsubseteq \mathfrak{M}_{1}$. Тогда по лемме 6 множество наследственных расслоенных подформаций $\mathfrak{F}$ с $b r$-направлением $\beta$, $\alpha \leqslant \beta \leqslant \gamma$, бесконечно, что противоречит лемме 5. Следовательно, $K(\mathfrak{F}) \cap \mathfrak{A}=K\left(\mathfrak{M}_{1}\right)$.

Пусть $t>2$. Обозначим $\mathfrak{M}=\mathfrak{M}_{1} \mathfrak{M}_{2}$ и $\mathfrak{H}=\mathfrak{M}_{3} \ldots \mathfrak{M}_{t}$. Если $|\triangle(\mathfrak{M})|=1$, то либо $K(\mathfrak{M})=(A)$, где $A$ - простая неабелева группа, либо $K(\mathfrak{M})=\left(Z_{p}\right)$ для некоторого простого числа $p$. В последнем случае $\mathfrak{M}=\mathfrak{N}_{p}$, что противоречит несократимости факторизации $\mathfrak{F}$ и следствию 3.3 .7 [3]. Следовательно, либо $K(\mathfrak{M})=(A)$ для простой неабелевой групшы $A$, либо $|\triangle(\mathfrak{M})|>1$. В любом случае $|\pi(\mathfrak{M})|>1$. Значит, по лемме 3.2.5 [3] $\mathfrak{M} \nsubseteq \mathfrak{N}$. Тогда по теореме 1 формация $\mathfrak{H}$ абелева и в силу леммы 3.2 .5 [3] следует, что $t=3$. Утверждение 1 ) доказано.

Утверждение 2) непосредственно следует из теоремы 1.

Пусть $t=3$. Допустим, что $\mathfrak{M}_{1} \nsubseteq \mathfrak{N}$. Тогда $\left|\pi\left(\mathfrak{M}_{1}\right)\right|>1$ и по теореме $1 \mathfrak{M}_{2} \mathfrak{M}_{3}$ - абелева формация. В этом случае $\mathfrak{M}_{2} \mathfrak{M}_{3}$ ограничена по параметру $c$, что противоречит лемме 3.2.5 [3]. Следовательно, $\mathfrak{M}_{1}$ нильпотентна. По лемме $7 \mathfrak{M}_{1} \mathfrak{M}_{2}-$ расслоенная формация с направлением $\beta$. Следовательно, по теореме $1 \mathfrak{M}_{1}$ и $\mathfrak{M}_{1} \mathfrak{M}_{2}-$ метанильпотентные однопорожденные расслоенные формации. Кроме того, так как $\left|\pi\left(\mathfrak{M}_{1} \mathfrak{M}_{2}\right)\right|>1$, то по лемме 3.2 .5 [3] $\mathfrak{M}_{1} \mathfrak{M}_{2}$ ненильпотентна. И значит, по теореме $1 \mathfrak{M}_{3}$ - однопорожденная абелева формация.

Покажем, что $\mathfrak{M}_{2}$ - нильпотентная формация. Пусть $m_{1}-$ минимальный спутник формации $\mathfrak{M}_{1} \mathfrak{M}_{2}$. По теореме 5 и следствию 5.1 работы [5] $m_{1}\left(Z_{p}\right) \subseteq \mathfrak{N}$ для всех $Z_{p} \in$ $K\left(\mathfrak{M}_{1} \mathfrak{M}_{2}\right)$. Поскольку формация $\mathfrak{F}$ разрешима и $K(\mathfrak{F})=K\left(\mathfrak{M}_{1}\right)$, то $\left|\pi\left(\mathfrak{M}_{1}\right)\right|>1$. Обо- 
значим через $\mathfrak{X}=\bigcup_{p \in \pi\left(\mathfrak{M}_{1}\right)} m_{1}\left(Z_{p}\right)$. Предположим, что $\mathfrak{M}_{2} \nsubseteq R_{0}(\mathfrak{X})$. Пусть $A-$ группа минимального порядка из $\mathfrak{M}_{2} \backslash R_{0}(\mathfrak{X})$. Тогда $A$ - монолитическая групша с монолитом $R$. Поскольку $\mathfrak{M}_{1} \mathfrak{M}_{2}$ - метанильпотентная формация, то $R$ - элементарная абелева $p$-группа для некоторого простого числа $p$. Пусть $q \in \pi\left(\mathfrak{M}_{1}\right) \backslash\{p\}$ и $T=Z_{q}$ (A. Поскольку $O_{q}(A)=1$, то ввиду леммы $2[9] T_{\gamma\left(Z_{q}\right)}=K$, где $K$ - база регулярного сплетения $T$. Кроме того, $T_{\alpha\left(Z_{q}\right)}=K$. Но $T / K \cong A \in \mathfrak{M}_{2}$. Тогда $T \in \mathfrak{N}_{q} \mathfrak{M}_{2} \subseteq \mathfrak{M}_{1} \mathfrak{M}_{2}$. Следовательно, $T / T_{\beta\left(Z_{q}\right)}=T / K \cong A \in m_{1}\left(Z_{q}\right)$. Противоречие. Значит $\mathfrak{M}_{2} \subseteq R_{0}(\mathfrak{X}) \subseteq \mathfrak{N}$.

Поскольку $\left|\pi\left(\mathfrak{M}_{1}\right)\right|>1$, по теореме 1 формации $\mathfrak{M}_{2}$ и $\mathfrak{M}_{2} \mathfrak{M}_{3}$ однопорождены.

Пусть $B \in R_{0}(\mathfrak{X})$. Но по теореме $1 \pi\left(m_{1}(L)\right) \cap \pi\left(\mathfrak{M}_{3}\right)=\varnothing$ для всех $L \in K\left(\mathfrak{M}_{1} \mathfrak{M}_{2}\right)$. Следовательно, $\pi(B) \cap \pi\left(\mathfrak{M}_{3}\right)=\varnothing$. Значит $\pi\left(\mathfrak{M}_{2}\right) \cap \pi\left(\mathfrak{M}_{3}\right)=\varnothing$. Необходимость доказана.

Достаточность. Пусть $\mathfrak{F}=\mathfrak{M}_{1} \ldots \mathfrak{M}_{t}$, где $\mathfrak{M}_{1}, \ldots, \mathfrak{M}_{t}$ - неединичные формации, удовлетворяющие условиям 1$)-4)$. При $t=2$ по пункту 2) и теореме $1 \mathfrak{F}-$ однопорожденная расслоенная формация. Рассмотрим случай при $t=3$. Если $\mathfrak{M}_{1}=\mathfrak{N}_{p}$, то $\mathfrak{M}_{2} \subseteq \mathfrak{N}_{p}$ и $\mathfrak{M}_{3} \subseteq \mathfrak{N}_{p}$. Противоречие с несократимостью факторизации $\mathfrak{F}$. Поэтому $\left|\pi\left(\mathfrak{M}_{1}\right)\right|>1$. Поскольку $\mathfrak{M}_{1} \subseteq \mathfrak{N}$, то $m(A)=(1)$ для любой групшы $A \in K\left(\mathfrak{M}_{1}\right)$, где $m$ - минимальный спутник формации $\mathfrak{M}_{1}$. Значит, $\pi\left(\mathfrak{M}_{2} \mathfrak{M}_{3}\right) \cap \pi(m(A))=\varnothing$ для всех $A \in K\left(\mathfrak{M}_{1}\right)$. Видим, что выполняются все условия теоремы 1 . Таким образом, $\mathfrak{F}$ - однопорожденная расслоенная формация с направлением $\beta$. Теорема доказана.

СлЕДСТВИЕ 4. Пусть $\mathfrak{F}=\mathfrak{M}_{1} \ldots \mathfrak{M}_{t}$ - несократимая факторизация биканонической (композичионной) формачии $\mathfrak{F}$, причем формачия $\mathfrak{M}_{1}$ также является биканонической (композичионной). Формачия $\mathfrak{F}$ тогда и только тогда однопорохсдена, когда выполняются следующие условия:

1) $t \leqslant 3, K(\mathfrak{F}) \cap \mathfrak{A}=K\left(\mathfrak{M}_{1}\right) u|\triangle(\mathfrak{F})|>1$;

2) при $t=2$ выполняются следующие пункты:

- $\mathfrak{M}_{1}$ - метанильпотентная однопорожденная биканоническая (композичионная) формаиия;

- $\pi\left(\mathfrak{M}_{2}\right) \cap \pi(m(B))=\varnothing \partial л я$ всех $B \in K\left(\mathfrak{M}_{1}\right)$, әде $m-$ минимальный спутник формачии $\mathfrak{M}_{1}$

- если $\left|\pi\left(\mathfrak{M}_{1}\right)\right|>1$, то $\mathfrak{M}_{2}$ - однопорожденная формачия, причем $\mathfrak{M}_{1} \nsubseteq \mathfrak{N}$ влечет $\mathfrak{M}_{2} \subseteq \mathfrak{A}$

- если $\left|\pi\left(\mathfrak{M}_{1}\right)\right|=1$, то $\mathfrak{M}_{2} / O_{p}\left(\mathfrak{M}_{2}\right)$ - однопорожденная формачия;

3) если $t=3$, то $\mathfrak{M}_{1}$ и $\mathfrak{M}_{1} \mathfrak{M}_{2}$ - однопорожденные биканонические (композичионные) формачии, $\mathfrak{M}_{2}, \mathfrak{M}_{3}, \mathfrak{M}_{2} \mathfrak{M}_{3}$ однопорохсдены, причем формаиии $\mathfrak{M}_{1}, \mathfrak{M}_{2}$ нильпотентны, формачия $\mathfrak{M}_{3}$ абелева $и \pi\left(\mathfrak{M}_{2}\right) \cap \pi\left(\mathfrak{M}_{3}\right)=\varnothing$.

В процессе доказательства теоремы было установлено, имеющее самостоятельный интерес

СлЕдСТВИЕ 5. Пусть $\mathfrak{F}=\mathfrak{M}_{1} \ldots \mathfrak{M}_{t}$ - несократимая факторизачия однопорожсденной расслоенной формации $\mathfrak{F}$ с br-направлением $\beta, \alpha \leqslant \beta \leqslant \gamma$, причем формаиия $\mathfrak{M}_{1}$ так же расслоенная с направлением $\beta$. Если $\mathfrak{M}_{1}$ - ненильпотентная формачия, то $t=2$.

ЛЕмма 16. Пусть $\mathfrak{F}=\mathfrak{N}_{\pi} \mathfrak{H}-$ расслоенная формация $с$ направлением $\beta, \alpha \leqslant$ $\beta \leqslant \gamma$, причем $|\pi|>1$. Тогда $\mathfrak{H}=R_{0}\left(\bigcup_{A \in K(\mathfrak{F})} f(A)\right)$, әде $f-$ минимальный спутник формачии $\mathfrak{F}$. 
ДокАЗАТЕЛЬСТво. Обозначим $\mathfrak{X}=\bigcup_{A \in K(\mathfrak{F})} f(A)$. Предположим, что $\mathfrak{H} \nsubseteq R_{0}(\mathfrak{X})$ и пусть $G$ - группа минимального порядка из $\mathfrak{H} \backslash R_{0}(\mathfrak{X})$. Тогда $G$ - монолитическая группа с монолитом $R$. Поскольку $\mathfrak{H} \subseteq \mathfrak{F}$, то $G \in \mathfrak{F}$. Если $R$ - неабелева группа и $K(R)=(B)$, то $G / O_{B^{\prime}}(G) \cong G \in f(B) \subseteq R_{0}(\mathfrak{X})$. Противоречие. Значит $R$ - элементарная абелева $p$-группа для некоторого $p \in \mathbb{P}$. Рассмотрим регулярное сплетение $T=Z_{q} \imath G=[K] G$, где $q \in \pi \backslash\{p\}$, а $K$ - база сплетения. Поскольку $O_{q}(G)=1$, по лемме $2[9] T_{\gamma\left(Z_{q}\right)}=O_{Z_{q}}(T)=K=T_{\alpha\left(Z_{q}\right)}=T_{\beta\left(Z_{q}\right)}$. Так как $T / K \cong G \in \mathfrak{H}$, то $T \in \mathfrak{N}_{q} \mathfrak{H} \subseteq \mathfrak{N}_{\pi} \mathfrak{H}=\mathfrak{F}$. Следовательно, $T / T_{\beta\left(Z_{q}\right)}=T / K \cong G \in f\left(Z_{q}\right) \subseteq R_{0}(\mathfrak{X})$. Получили противоречие. Поэтому $\mathfrak{H} \subseteq R_{0}(\mathfrak{X})$.

Покажем обратное включение. Ввиду теоремы $5[5] f(B)=\operatorname{form}\left(G / G_{\beta(B)} \mid G \in\right.$ $\mathfrak{F})$ для всех $B \in K(\mathfrak{F})$. Пусть $M-$ произвольная группа из $\mathfrak{F}$. Если $B \notin K(M)$, то $M / M_{\beta(B)} \cong 1 \in \mathfrak{H}$. Пусть $B \in K(M)$. Поскольку $M^{\mathfrak{H}} \subseteq \mathfrak{N}_{\pi}$, то $M^{\mathfrak{H}} \subseteq F(M) \subseteq$ $F_{B}(M)$. В случае, когда $B \notin \mathfrak{A}, M / M_{\beta(B)}=M / F_{B}(M) \in \mathfrak{H}$. Пусть $B \in \mathfrak{A}$. Из нильпотентности $M^{\mathfrak{H}}$ заключаем, что $M^{\mathfrak{H}} \subseteq O_{B^{\prime}, B}(M)=M_{\alpha(B)} \subseteq M_{\beta(B)}$. Таким образом, $M / M_{\beta(B)} \in \mathfrak{H}$ для любой группы $M$ из $\mathfrak{F}$. Значит, $f(B) \subseteq \mathfrak{H}$. В силу произвольности $B$ можно сделать вывод, что $R_{0}(\mathfrak{X}) \subseteq \mathfrak{H}$. Следовательно, $\mathfrak{H}=R_{0}(\mathfrak{X})$. Лемма доказана.

ЛЕмма 17. Пусть $\mathfrak{F}=\mathfrak{N}_{p} \mathfrak{H}-$ несократимая факторизация однопорожденной расслоенной формачии $\mathfrak{F}$ с br-направлением $\beta, \alpha \leqslant \beta \leqslant \gamma$. Тогда $\mathfrak{H} / O_{p}(\mathfrak{H})=$ $R_{0}\left(\bigcup_{A \in K(\mathfrak{F})} f(A)\right)$, әде $f-$ минимальный спутник формации $\mathfrak{F}$.

ДокаЗАтЕЛЬство. Пусть $\mathfrak{X}=\bigcup_{A \in K(\mathfrak{F})} f(A)$. По теореме $1 K(\mathfrak{H}) \cap \mathfrak{A} \subseteq\left(Z_{p}\right)$. Тогда по лемме 7 формация $\mathfrak{F}$ обладает спутником $h$ таким, что $h(A)=\mathfrak{H}$ для всех $A \in$ $K(\mathfrak{F})$. Пусть $H$ - произвольная группа из $\mathfrak{H}$. Тогда $H / O_{p}(H) \in \mathfrak{H}=h\left(Z_{p}\right)$. Поскольку $O_{p}\left(H / O_{p}(H)\right)=1$, по лемме $13 H / O_{p}(H) \in f\left(Z_{p}\right)$. Значит, $\mathfrak{H} / O_{p}(\mathfrak{H}) \subseteq R_{0}(\mathfrak{X})$.

Докажем обратное включение. Несложно показать, что $\mathfrak{F}=\mathfrak{N}_{p} \mathfrak{H}=\mathfrak{N}_{p}\left(\mathfrak{H} / O_{p}(\mathfrak{H})\right)$. Пусть произвольная группа $M \in \mathfrak{F}=\mathfrak{N}_{p}\left(\mathfrak{H} / O_{p}(\mathfrak{H})\right)$. В этом случае $M^{\mathfrak{H} / O_{p}(\mathfrak{H})} \in \mathfrak{N}_{p}$, т.е. $M^{\mathfrak{H} / O_{p}(\mathfrak{H})} \subseteq F(M) \subseteq F_{B}(M)$ для любого $B \in K(M)$. Ввиду теоремы $5[5] f(B)=$ $\operatorname{form}\left(G / G_{\beta(B)} \mid G \in \mathfrak{F}\right)$ для всех $B \in K(\mathfrak{F})$. Если $B \notin K(M)$, то $M / M_{\beta(B)} \cong 1 \in$ $\mathfrak{H}$. Пусть $B \in K(M)$. В случае, когда $B \notin \mathfrak{A}, M / M_{\beta(B)}=M / F_{B}(M) \in \mathfrak{H} / O_{p}(\mathfrak{H})$. Пусть $B \in \mathfrak{A}$. Из нильпотентности $M^{\mathfrak{H} / O_{p}(\mathfrak{H})}$ заключаем, что $M^{\mathfrak{H} / O_{p}(\mathfrak{H})} \subseteq O_{B^{\prime},{ }_{B}}(M)$ $=M_{\alpha(B)} \subseteq M_{\beta(B)}$. Таким образом, $M / M_{\beta(B)} \in \mathfrak{H} / O_{p}(\mathfrak{H})$ для любой групшы $M$ из $\mathfrak{F}$. Значит, $f(B) \subseteq \mathfrak{H} / O_{p}(\mathfrak{H})$. В силу произвольности $B$ можно сделать вьвод, что $R_{0}(\mathfrak{X}) \subseteq$ $\mathfrak{H} / O_{p}(\mathfrak{H})$. Тогда $\mathfrak{H} / O_{p}(\mathfrak{H})=R_{0}(\mathfrak{X})$. Лемма доказана.

ТЕОРема 3. Пусть однопорожденная расслоенная формачия $\mathfrak{F}$ с br-направлением $\beta, \alpha \leqslant \beta \leqslant \gamma$, допускает несократимую факторизацию $\mathfrak{F}=\mathfrak{F}_{1} \mathfrak{F}_{2} \ldots \mathfrak{F}_{t}$, әде $\mathfrak{F}_{1}, \mathfrak{F}_{2}, \ldots, \mathfrak{F}_{t}-$ неразложимые формачии, в которой формаиия $\mathfrak{F}_{1}$ также является расслоенной с направлением $\beta$. Если $t \neq 2$ или формация $\mathfrak{F}_{1}$ не является примарной, то все множители факторизачии $\mathfrak{F}$ однозначно определены. $B$ случае $\mathfrak{F}=\mathfrak{N}_{p} \mathfrak{F}_{2}$ однозначно определена формачия $\mathfrak{F}_{2} / O_{p}\left(\mathfrak{F}_{2}\right)$.

ДокАЗАТЕЛЬСТво. Предположим, что формация $\mathfrak{F}$ допускает две различные несократимые факторизации $\mathfrak{F}=\mathfrak{M}_{1} \mathfrak{M}_{2} \ldots \mathfrak{M}_{t}=\mathfrak{H}_{1} \mathfrak{H}_{2} \ldots \mathfrak{H}_{r}$ с неразложимыми множителями, в которых $\mathfrak{M}_{1}$ и $\mathfrak{H}_{1}-$ расслоенные формации с направлением $\beta$.

По теореме $2 t \leqslant 3$ и $r \leqslant 3$. Если $t$ или $r$ равны 3 , то формация $\mathfrak{F}$ разрешима, а следовательно, локальна. Единственность разложения однопорожденной локальной формации доказана в теореме 3.4.1 [3]. Осталось рассмотреть случай $t=r=2$. 
Пусть $\mathfrak{F}=\mathfrak{M}_{1} \mathfrak{M}_{2}=\mathfrak{H}_{1} \mathfrak{H}_{2}$. Если хотя бы одна из формаций $\mathfrak{M}_{1}$ или $\mathfrak{H}_{1}$ не нильпотентна, то по теореме 1 формация $\mathfrak{F}$ разрешима. Значит, остается вариант, когда обе формации $\mathfrak{M}_{1}$ и $\mathfrak{H}_{1}$ нильпотентны. Кроме того, по теореме $2 K\left(\mathfrak{M}_{1}\right)=K\left(\mathfrak{M}_{1}\right) \cap \mathfrak{A}=$ $K(\mathfrak{F}) \cap \mathfrak{A}=K\left(\mathfrak{H}_{1}\right)$. Тогда $\mathfrak{F}=\mathfrak{N}_{\pi} \mathfrak{M}_{2}=\mathfrak{N}_{\pi} \mathfrak{H}_{2}$, где $\pi=\pi\left(\mathfrak{M}_{1}\right)=\pi\left(\mathfrak{H}_{1}\right)$. Из лемм 16 и 17 заключаем, что если $|\pi|>1$, то $\mathfrak{M}_{2}=\mathfrak{H}_{2}$, а если $|\pi|=1$, то $\mathfrak{M}_{2} / O_{p}\left(\mathfrak{M}_{2}\right)=\mathfrak{H}_{2} / O_{p}\left(\mathfrak{H}_{2}\right)$. Теорема доказана.

СлЕДСТВИЕ 6. Пусть однопорожденная биканоническая (композиционная) формация $\mathfrak{F}$ допускает несократимую факторизацию $\mathfrak{F}=\mathfrak{F}_{1} \mathfrak{F}_{2} \ldots \mathfrak{F}_{t}$, дде $\mathfrak{F}_{1}, \mathfrak{F}_{2}, \ldots, \mathfrak{F}_{t}$ неразложимые формачии, в которой формачия $\mathfrak{F}_{1}$ также является биканонической (композичионной). Если $t \neq 2$ или формация $\mathfrak{F}_{1}$ не является примарной, то все множители факторизачии $\mathfrak{F}$ однозначно определены. $B$ случае $\mathfrak{F}=\mathfrak{N}_{p} \mathfrak{F}_{2}$ однозначно определена формация $\mathfrak{F}_{2} / O_{p}\left(\mathfrak{F}_{2}\right)$.

\section{СПИСОК ЦИТИРОВАННОЙ ЛИТЕРАТУРЫ}

[1] Скиба А. Н. О факторизациях одного класса формаций конечньх групп // Вопросы алгебры. Вып. 7. Гомель. 1992. С. 108-110.

[2] Skiba A. N. On nontrivial factorizations of an onegenerated local formation of finite groups // Proc. Int. Conf. Algebra Dedicat. Mem. A. I. Malcev (Novosibirsk, 21-26 August 1989). Novosibirsk, 1989. P. 111.

[3] Скиба А. Н. Алгебра формаций. Мн.: Беларуская навука, 1997.

[4] Vedernikov V.A. Maximal satellites of $\Omega$-foliated formations and Fitting classes // Tp. МИАН. 2. 2001. V. 2. Р. 217-233.

[5] Ведерников В.А., Сорокина М.M. $\Omega$-расслоенные формации и классы Фиттинга // Дискретная матем. 2001. Т. 13. № 3. С. 125-144.

[6] Шеметков Л.А., Скиба А. Н. Формации алгебраических систем. М.: Наука, 1989.

[7] Скиба А. Н., Шеметков Л. А. Кратно $\omega$-локальные формации и классы Фиттинга конечных групп // Матем. труды. 1999. Т. 2. № 2. С. 114-147.

[8] Doerk K., Hawkes T. Finite Soluble Groups. Berlin-New York: Walter de Gruyter, 1992.

[9] Скиба А.Н., Шеметков Л. А. О минимальном композиционном экране композиционной формации // Вопросы алгебры. Вып. 7. Гомель. 1992. С. 39-43.

Брянский государственный университет им. И. Г. Петровского

Поступило

E-mail: elovikovab@mail.ru

02.04 .2002 